\title{
A DEVELOPMENT FRAMEWORK FOR A CONVERSATional Agent to Explore MACHINE LEARNING CONCEPTS
}

\author{
Ayse Kok Arslan \\ Oxford Alumni of Northern California, Santa Clara, USA
}

\begin{abstract}
This study aims to introduce a discussion platform and curriculum designed to help people understand how machines learn. Research shows how to train an agent through dialogue and understand how information is represented using visualization. This paper starts by providing a comprehensive definition of AI literacy based on existing research and integrates a wide range of different subject documents into a set of key AI literacy skills to develop a user-centered AI. This functionality and structural considerations are organized into a conceptual framework based on the literature. Contributions to this paper can be used to initiate discussion and guide future research on AI learning within the computer science community.
\end{abstract}

\section{KEYWORDS}

Machine learning, visual editing, construction, neural nets, artificial intelligence.

\section{INTRODUCTION}

There is a need to add research that investigates what new skills will be needed in the future as AI transforms the way people communicate, work, and live with each other and technically. It is seen as a technological innovation to transform our society, economy and jobs in the digital community, let alone its accelerated outcome during the Covid phase. Some of the most wellknown examples of AI non-driving vehicles, voice assistants, online search engines, robotic vendors, etc. Despite the destructive AI myth represented in films like Terminator or I, Robot, the fact is that nowadays clever algorithms contain a series of simple rules applied to a great series of numbers, and the result is called AI.

This study examines the use of a chat agent with four consecutive AI modules, as well as testing machine learning and information representation. This study provides a design framework for a program related to training content. While previous knowledge of chat agents and other factors can affect a user's ability to understand, the basic assumption is that all users can benefit greatly from the interaction with the agent and chat. Within this context, the following ideas are based on:

1. Collaborating with the agent helps to increase participation in the whole process.

2. Agent recognition and training content lead to user learning and understanding of how machines learn. 


\section{RELATED WORK}

Historically, computer use refers to mainframe computers for editing and writing hidden lines of text code that can be made. Learning a low-level programming language, such as BASIC established in 1964, meant remembering instructions and working in a solid text environment without feedback or help. That learning to think like a software engineer can be made easier and more playable is proved by Papert, who had the original idea of the Logo program in 1967 and made a line for Scratch's continued success [1].

When it comes to integrating CS into the curriculum, today's efforts largely incorporate the use of ML-based language-enabled language through platforms such as the Mechanized Learning for Kids [4] website and the Cognimates project [12 involving blocks that provide easy access of various cloud AI services. This does not explicitly provide a plan for building neural networks connectors dealing with the programs they offer for training and using neural networks only.

To give some examples, Google's Teachable Machine [4] provides a web page where users can train the image classification system while TensorFlow provides a playground to collaborate, train, and test an in-depth neural learning net.

- Learning with AI: While most AI educational programs are the latest in a long line of ideas, the idea of introducing people to AI ideas began to work with Seymour Papert and Cynthia Solomon using the LOGO program and the Turtle robot $[3,5,7,9]$. It has served as the basis for much of the current work. Many platforms teach AI by having an individual program in block-based languages including Cognimates [23], Machine Learning for Kids [29, 17] and eCraft2Learn [27]. Other platforms introduce AI within the context of robots, such as Popbots [19, 21], as well as performance enhancements [3] and MIT App Inventor AI Extensions [13, 17, 19].

- Learning with Conversational Agents: Conversation agents are also used for learning, often as intelligent educators and learning friends [5]. The structure of these communication systems varies greatly, from incorporations and results based on the text [6] to integrated agents that can express emotions [7].

Researchers exploring how to engage younger students in design activities involving AI [4] identified five "major ideas" of AI to guide the development of standards:

1) "Computers detect the earth using sensors";

2) "Agents maintain models / representation of land and use it for consultation";

3) "Computers can learn from data";

4) "Making social media agents is a major challenge for AI engineers"; and

5) "AI applications can affect society in both positive and negative ways" [13]].

The model development starts with the following questions about AI:

What is AI?

Explaining what AI can confuse even experts $[16,12]$, as the term has changed over the years. Nilson describes AI as "that work dedicated to making machines smarter ... [where] intelligence is the quality that makes a business more efficient and foresight" [10]. However, Schank notes that the definitions of intelligence may vary depending on the investigator and their approach to understanding AI [16]. He suggests that there are two main objectives of AI research - "building a smart machine" and "finding a kind of intelligence" [16]. He then proposed a set of features that 
included common "intelligence" - communication, knowledge of the world, internal knowledge, purpose, and art - emphasizing that the ability to read is the most important factor in intelligence [16].

\section{What Can AI Do?}

While AI has been able to find patterns in the amount of data, perform repetitive tasks, and make decisions in controlled environments, people now live better in many tasks that require art, emotion, information transfer, and social interaction.

How does AI work?

A better understanding of how AI works can help people build more sensible types of programs they work with. For this and other reasons, much of the research available on AI education in university and K-12 environments focuses on informing how AI works.

\section{Cognitive Systems}

Cognitive systems - or AI systems are rated after ideas about the human mind [4] - are used in a variety of application domains, including WordNet, IBM's Watson, expert systems, and cognitive educators. Most comprehension program messages cover topics related to information representation, planning, decision making, problem solving and learning.

Cognitive systems use many techniques for planning, decision-making, problem-solving and learning. Users may not need to understand all of these strategies in detail, but a higher understanding of how computers make decisions can help in interpreting and understanding the algorithms [29].

\section{Machine Learning}

Many students think that computers think like humans and want to make connections between human perceptions of understanding and machine learning [12]. Students are also often surprised that ML requires human decision-making and is not self-inflicted.

Research suggests that one of the ways to eliminate students' misconceptions about ML is to get involved in integrated integration. Sulmont et al. while others suggest that students develop physical algorithms to understand them in a practical way $[4,6,12]$. This technique has also been used in CS education [2]. In general, AI manual testing has been used as a means of implementing a variety of AI education programs (e.g. [4]), including projects where students can train ML models to analyze their movements and gestures [4,14].

\section{Robotics}

Understanding that AI agents can physically act on and react to the world is an important prerequisite for understanding robotics. Learning about sensors and their capabilities (one of the "big ideas" of AI [13]) can also aid in understanding how AI devices gather data and interface with the world.

This study provides the development of a platform and curriculum around the three "Great Ideas" in AI, as it directs ideas that have a major impact on making people understand about AI [3]. At the same time, these ideas also allow people to explore the use of a visual chat interface. The three ideas are: 
- Representation and Consultation: People are expected to understand how the agent learns and represents new information. The agent generates two different visuals to indicate the representation of the information.

- Conceptualization: The agent also demonstrates the concept of how machines classify ideas. Individuals witness instances when the agent might succeed or fail in its learning and make attempts to correct it.

- Social Impact: The curriculum emphasizes the moral and social impact of the AI community through structured dialogue and reflection on the impacts of larger image environments.

Agent platform is a visual web connector that can be linked to a web browser and people engage with the agent in a chat in a small setting led by a personal facilitator.

\section{Proposed Model}

This study examines the use of a chat agent with four consecutive AI modules, as well as testing machine learning and information representation.

\subsection{Overview of AI Modules}

The AI curriculum consists of four modules: "Do You Know the Agent?", "Teaching a Lawyer", "Machine Witnessing", and "AI and Ethics". Before entering the first module, individuals learn to communicate with the agent through an introduction, where the agent greets and talks with them.

Part 1, "What Does the Agent Know?" introduces people to represent information and consultation using mind maps. The representation method used is a mind map or "mind map", which people can analyse to find relevant attributes. Positive attributes (existing) are shown as blue circles, and negative (missing) circles are shown as red circles. Users can also analyze the corpus given by the agent to create mind maps, and thus draw connections between natural language sentences and related mind map details.

In the next module, Module 2, "Teaching Agent", individuals are assigned the task of providing the agent with data on selected subjects. People can give the agent any information about a topic they would like to find. This enables them to put AI learning experience into their knowledge and interests. Agent works as an AI with minimal knowledgeable information, and users help an agent create mind maps on each topic. The concept map concept is presented in Module 1 and is based on the following modules.

Module 3: "Machine learning", is where users look at the learning process and the agent's thinking. They ask the agent to make a guess based on a previously taught concept and the agent calculates the similarity of the words in each concept by using words that represent the words representing the agent ideas and showing these schools using a bar graph. It is important for users to understand why and why the agent may be incorrectly guessing by drawing a link between similarities within Module 2 mind maps and Module 3 scores, and how to use this link.

In the final module, Module 4, "AI and Ethics", facilitators lead a discussion on how data negotiating agents and data-learning agents are used in society with both positive and negative outcomes. These discussion questions are divided into the Learning Resources section. Users can think of situations where the agent makes mistakes, and then be asked questions such as, "Will the agent know if what we are teaching is right or not?" and "How would you feel if an agent 
found out something wrong with you?". The purpose of this section is to empower users with AI design tools with ethical principles. The study also aims to test users on the social consequences of mistakes made by AI, and how they can reduce injuries (Payne 2019).

\subsection{System Design}

The system is designed to be easy to use. It can be used using any browser anywhere you have internet access. The main features of the program are (1) a speech synthesizer, (2) a speech identifier, (3) a semantic parser, (4) a word map classifier, and (5) a website visualizer, as follows:

1. Speech amplifier: This section includes the voice of the agent using the Web Speech API. It should be noted that a particular word from the visual interface of speech recognition should be chosen so that it is not as gender neutral as possible. (A common advice is to refer to the agent as "it" instead of "he" or "she".)

2. Speech identifier: This section converts user speech to text using the Web Speech API.

3. Semantic parser: This section performs natural language processing using NLTK $[29,32,17$, 18] and CoreNLP tools (Manning et al. 2014). The NLTK toolkit works with words, while the CoreNLP toolkit renders parts of speech [30] and processes sentences obtained from speech recognition. The adjective performs the following three functions used in each function.

a) Name identification: As a job introduction, the agent asks people for their name and location. The relay conveys the input received after each query, removing all nouns, proper nouns, and foreign names. The specified name or location is the appropriate last name seen.

b) Subject Identification: In the first module, the tester identifies which user the question is asking. Since the number of ideas that the agent is aware of is limited, the examiner searches for these ideas. When a familiar concept is found, the parser retrieves the identified concept and a pre-programmed mind map is displayed to users. These mind maps are created offline using a third-party explorer capability.

c) Mind Map Builder: In Modules 1 and 2, the agent demonstrates his / her knowledge of ideas and words on mind maps that are generated according to sentence structure and speech components. An adjective identifies a field of concept for the interaction between descriptive words and a topic (e.g., a negative interaction between water and desert), and then sends this information to a web browser.

4. Classifier: This section classifies the word by comparing word representation using NLTK's Wordline Interface (Loper and Bird 2002; Fellbaum 1998). In each sense, all the descriptive words are compared to each other. Once the similarity between all pairs has been calculated, the overall similarity between a concept and topic is its weighted average similarity score. The classifier returns a normalized score for each topic, denoting the topic with the highest similarity score to be the topic that the concept relates to.

5. Visualizer: This section creates mind maps and histograms based on pre-defined sentences and user-defined sentences using D3.js [15, 25].

To show how the parts fit together, in the introduction, the system prompts the user to speak; sends a user voice response to a browser-based speech recognition, which converts the response into text; stores text response to user location session data; sends a text response to a server-based 
server, processing the text for important details; returns the processed information to a browserbased speech compiler, which causes the agent to speak, causing the user to speak again; and repeats the process until it ends the conversation.

Figure 1. includes a view of the histogram, which shows similarities between the concept and the sentence of the title.

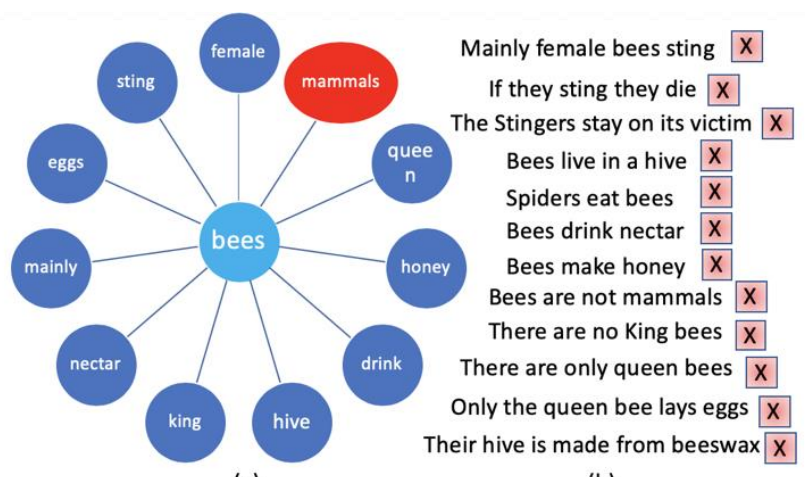

(a)

(b)

Figure 1: (a) Mind map composed of sentences in (b). This is shown in Module 2 on the agent's website. (b) Users of sentences have informed the agent during the study.

\section{RESUlTS AND DISCUSSION}

To measure system performance, a small pilot study can be done to determine if people understand the visual representations. Once hired people can hear about the three different perspectives on information representation (Modules 1 and 2) and the three different machine learning perspectives (Module 3) shown in Figure 2.

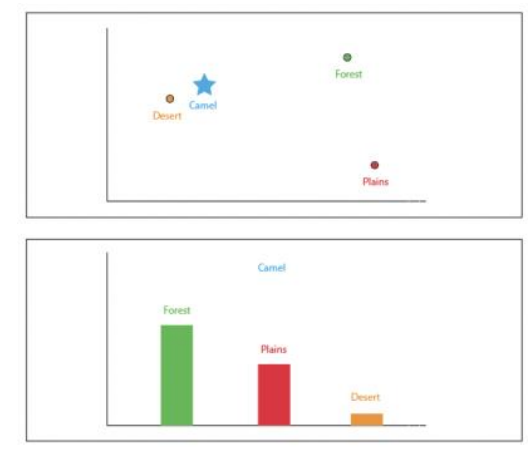

Figure 2: As shown in the orange bar in this histogram view, the agent can guess which concept will affect it.

Testing can also be done even if the knowledge of the learning platform and the content of the training improves people's understanding of how machines represent information and learn. Specifically, the level of user engagement can be assessed by measuring the number of sentences used in the conversation with the agent, and whether it corresponds to their level of understanding.

A consistent test protocol can be followed at different times. The size of the session can be from one to four, each with a facilitator. First, researchers can randomly test participants' information 
on voice assistants such as Siri and Alexa via icebreaker. After this, they can conduct a prefollow-up test for the agent (Figure 3). Modules 1 to 4 must then be completed respectively. All work will last $\sim 60-80$ minutes depending on the length of the conversation. People should do all the tests individually on paper and without the slightest distraction from the investigators. In addition to the measurement data from the test, researchers can also perform video recordings of the time, participants in log sentences contribute and record users' responses to test questions.

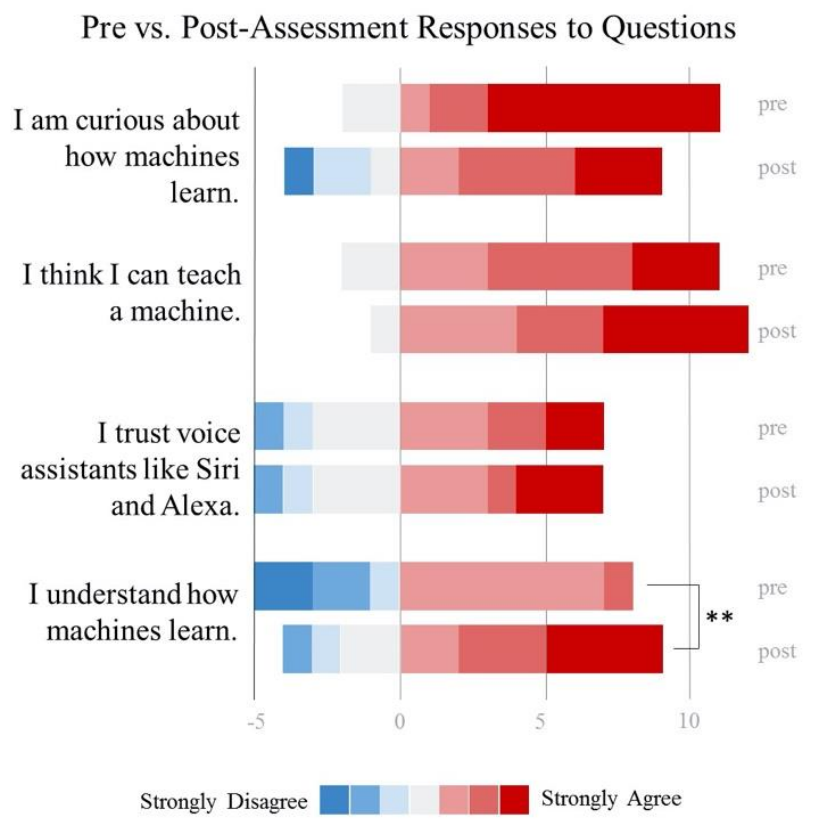

Figure 3: Sample responses to pre- vs. post-examination

\section{Assessments}

To answer the idea that people can learn effectively and to understand how machines learn, the following questions can be asked for assessment purposes:

1. What sentences can you say to the facilitator to create the following mind map in Figure 1? (This tests their understanding of how information is represented.)

2. What can you tell the agent about a particular name so that he can correctly guess the related topic? (This tests their understanding of how the agent reads.)

3. Why would an agent find it difficult to match another word to a particular topic even if he knows everything that needs to be known about them? (This is an open-ended questionnaire for examining potential mistakes an agent may make.)

4. Have you ever tried to mention the name 'agent' to the agent? If not, ask your tutor if you can try. Does the agent recognize its own name? If not, why not? Can you think of another name the agent would not see? (This is an open question in the agent's internal language management.)

These pre- and post-test assessments can also be used to assess individual self-awareness as an engineer and motivation to learn. People can be asked to rate how much they agree or disagree with the various statements on a scale of 1 to 5 . 
1. I want to know how a machine learns.

2. I think I can teach it to the machine.

3. I trust voice assistants like Siri and Alexa.

4. I understand how the machine learns.

5. The activities we have done today have been helpful in learning how machines learn. (Post-test only.)

6. The mind map of each concept and topic helped me think about the agent's brain. (Post-test only.)

7. The histogram helped me to understand how the agent made decisions. (Post-test only.)

\section{CONClusion AND Future Scope}

This work introduces a development framework for a conversational agency that educates individuals about the representation of information and machine learning.

By training an agent, recognizing his mistakes, and retraining an agent, individuals can make sense of the intelligence of a representative. In the future, the content of the agent training should be expanded to address more topics in AI. Hopefully, this work will promote more AI training content using conversational agents and viewing tools to help individuals understand the AI algorithms.

The current framework of research has some limitations. First, it includes a small number of participants. Further testing may reinforce the claim that the agent is operating. Also, there is a need to create a future iteration of this study in which researchers can compare the performance of the visual interface of another chat agent.

Computer scientists usually rely on statistical tools to demonstrate that a particular underlying factor had a "causal" effect on the outcome of interest. While in the natural sciences, causal effects are measured using lab experiments that can isolate the consequences of variations in physical conditions on the effect of interest, more often than not, social life (including education through means of AI) do not permit lab-like conditions that allow the effects of changes in the human condition to be precisely ascertained and measured. This would merely provide evidence on one of the causes, which may not even be one of the more important factors. In a quest for statistical "identification" of a causal effect, scientists might often have to resort to techniques that answer either a narrower or a somewhat different version of the question that motivated the research. So, research can rarely substitute for more complete works of synthesis, which consider a multitude of causes, weigh likely effects, and address spatial and temporal variation of causal mechanisms.

For this study to be conducted, there is a need to control how participants interact with the agent by reducing their knowledge required for participants to be able to train the agent in real time.

Last, but not least, the skills and design considerations outlined in this paper will need to be expanded to keep pace with new discoveries, technologies, and rapidly changing social norms. Researchers and educators of AI, and related technology and education communities should be encouraged to both participate in intimate discussions and design considerations in this paper and use them to lead and promote artistic and future research on AI learning. 


\section{REFERENCES}

[1] Agre, P. E. (1994). Surveillance and capture: Two models of privacy. The Information Society, 10(2), 101-127.

[2] Allen, J. (2016). Topologies of power. Beyond territory and networks. Routledge.

[3] Bratton, B. (2015). The Stack: On software and sovereignty. MIT Press.

[4] Bucher, T. (2018). If...then: Algorithmic power and politics. Oxford University Press.

[5] Castañeda, L., \& Selwyn, N. (2018). More than tools? Making sense of the ongoing digitizations of higher education. International Journal of Educational Technology in Higher Education, 15(1).

[6] Decuypere, M. (2019a). Open Education platforms: Theoretical ideas, digital operations and the figure of the open learner. European Educational Research Journal, 18(4), 439-460.

[7] Decuypere, M. (2019b). Researching educational apps: ecologies, technologies, subjectivities and learning regimes. Learning, Media and Technology, 44(4), 414-429.

[8] Decuypere, M. (2019c). STS in/as education: where do we stand and what is there (still) to gain? Some outlines for a future research agenda. Discourse: Studies in the Cultural Politics of Education, 40(1), 136-145

[9] Dieter, M., Gerlitz, C., Helmond, A., Tkacz, N., Vlist, F., Der, V., \& Weltevrede, E. (2018). Store, interface, package, connection : Methods and propositions for multi-situated app studies. CRC Media of Cooperation Working Paper Series No 4.

[10] Drucker, J. (2020). Visualization and Interpretation: Humanistic Approaches to Display. MIT Press. Journal of New Approaches in Educational Research, 10(1)

[11] Mathias, Decuypere The Topologies of Data Practices: A Methodological Introduction Fedorova, K. (2020). Tactics of Interfacing. Encoding Affect in Art and Technology. MIT Press. Goriunova, O. (2019). The Digital Subject: People as Data as Persons. Theory, Culture \& Society, 36(6), 125-145.

[12] Grommé F, Ruppert E. (2020). Population Geometries of Europe: The Topologies of Data Cubes and Grids. Science, Technology, \& Human Values, 45(2), 235-261.

[13] Gulson, K. N., Lewis, S., Lingard, B., Lubienski, C., Takayama, K., \& Webb, P. T. (2017). Policy mobilities and methodology: a proposition for inventive methods in education policy studies. Critical Studies in Education, 58(2), 224-241.

[14] Gulson, K. N., \& Sellar, S. (2019). Emerging data infrastructures and the new topologies of education policy. Environment and Planning D: Society and Space, 37, 350-366.

[15] Hartong, S. (2020). The power of relation-making: insights into the production and operation of digital school performance platforms in the US. Critical Studies in Education, 00(00), 1-16.

[16] Hartong, S., \& Förschler, A. (2019). Opening the black box of data-based school monitoring: Data infrastructures, flows and practices in state education agencies. Big Data \& Society, 6(1),

[17] Lash, S. (2012). Deforming the Figure: Topology and the Social Imaginary. Theory, Culture \& Society, 29(4-5), 261-287.

[18] Latour, B. (1986). Visualization and cognition: Thinking with eyes and hands. Knowledge \& Society, 6, 1-40. Retrieved from http://hci.ucsd.edu/10/readings/Latour(1986).pdf

[19] Law, J. (2004). After Method: Mess in Social Science Research. Psychology Press.

[20] Lewis, S. (2020). Providing a platform for "what works": Platform-based governance and the reshaping of teacher learning through the OECD's PISA4U. Comparative Education, 56(4).

[21] Lewis, S., \& Hardy, I. (2017). Tracking the Topological: The Effects of Standardised Data Upon Teachers' Practice. British Journal of Educational Studies, 65(2), 219-238.

[22] Light, B., Burgess, J., \& Duguay, S. (2018). The walkthrough method: An approach to the study of apps. New Media and Society, 20(3), 881-900.

[23] Lindh, M., \& Nolin, J. (2016). Information We Collect: Surveillance and Privacy in the Implementation of Google Apps for Education. European Educational Research Journal, 15(6), Lury, C., \& Day, S. (2019). Algorithmic Personalization as a Mode of Individuation. Theory, Culture \& Society, 36(2), 17-37.

[24] Mathias, Decuypere The Topologies of Data Practices: A Methodological Introduction Lury, C., Fensham, R., Heller-Nicholas, A., \& Lammes, S. (2018). Routledge Handbook of Interdisciplinary Research Methods. Routledge.

[25] Lury, C., Parisi, L., \& Terranova, T. (2012). Introduction: The Becoming Topological of Culture. Theory, Culture \& Society, 29(4-5), 3-35. 
[26] Lury, C., Tironi, M., \& Bernasconi, R. (2020). The Social Life of Methods as Epistemic Objects: Interview with Celia Lury. Diseña, 16, 32-55.

[27] Lury, C., \& Wakeford, N. (2012). Introduction: A perpetual inventory. Inventive Methods (pp. 1538). Routledge.

[28] Martin, L., \& Secor, A. J. (2014). Towards a post-mathematical topology. Progress in Human Geography, 38(3), 420-438.

[29] Piattoeva, N., \& Saari, A. (2020). Rubbing against data infrastructure(s): methodological explorations on working with(in) the impossibility of exteriority. Journal of Education Policy, 00(00), 1-21.

[30] Plantin, J. C., Lagoze, C., Edwards, P. N., \& Sandvig, C. (2018). Infrastructure studies meet platform studies in the age of Google and Facebook. New Media and Society, 20(1), 293-310.

[31] Prince, R. (2017). Local or global policy? Thinking about policy mobility with assemblage and topology. Area, 49(3), 335-341.

[32] Ratner, H. (2019). Topologies of Organization: Space in Continuous Deformation. Organization Studies, $1-18$.

[33] Ratner, H., \& Gad, C. (2019). Data warehousing organization: Infrastructural experimentation with educational governance. Organization, 26(4), 537-552.

[34] Ratner, H., \& Ruppert, E. (2019). Producing and projecting data: Aesthetic practices of government data portals. Big Data \& Society, 6(2), 1-16.

[35] Ruppert, E., Law, J., \& Savage, M. (2013). Reassembling Social Science Methods: The Challenge of Digital Devices. Theory, Culture \& Society, 30(4), 22-46.

[36] Suchman, L. (2012). Configuration. In C. Lury \& N. Wakeford (Eds.), Inventive Methods: The Happening of the Social (pp. 48-60). Taylor and Francis.

[37] Thompson, G., \& Cook, I. (2015). Becoming-topologies of education: deformations, networks and the database effect. Discourse: Studies in the Cultural Politics of Education, 36(5), 732-748.

[38] Thompson, G., \& Sellar, S. (2018). Datafication, testing events and the outside of thought. Learning, Media and Technology, 43(2), 139-151.

[39] van de Oudeweetering, K., \& Decuypere, M. (2019). Understanding openness through (in)visible platform boundaries: a topological study on MOOCs as multiplexes of spaces and times. International Journal of Educational Technology in Higher Education, 16(1).

[40] van de Oudeweetering, K., \& Decuypere, M. (2020). In between hyperboles: forms and formations in Open Education. Learning, Media and Technology, Advance online publication, 1-18.

[41] Williamson, B. (2017). Learning in the "platform society": Disassembling an educational data assemblage. Research in Education, 98(1), 59-82.

\section{AUTHORS}

Ayse received her MSc in Internet Studies in University of Oxford in 2006. She participated in various research projects for UN, Nato and the EU regarding HCI (human-computer interaction). She completed her doctorate degree in user experience design in Oxford while working as an adjunct faculty member at Bogazici University in her home town Istanbul. Ayse has also a degree in Tech Policy from Cambridge University. Currently, Ayse lives in Silicon Valley where she works as a visiting scholar for Google on human-computer interaction design.

(C) 2021 By AIRCC Publishing Corporation. This article is published under the Creative Commons Attribution (CC BY) license. 